\title{
Dynamic homogenization of composite and locally resonant flexural systems
}

\author{
René Pernas-Salomón and Gal Shmuel* \\ Faculty of Mechanical Engineering, Technion-Israel Institute of Technology, Haifa 32000, Israel
}

\begin{abstract}
Dynamic homogenization aims at describing the macroscopic characteristics of wave propagation in microstructured systems. Using a simple method, we derive frequency-dependent homogenized parameters that reproduce the exact dispersion relations of infinitely periodic flexural systems. Our scheme evades the need to calculate field variables at each point, yet capable of recovering them, if wanted. Through reflected energy analysis in scattering problems, we quantify the applicability of the homogenized approximation. We show that at low frequencies, our model replicates the transmission characteristics of semi-infinite and finite periodic media. We quantify the decline in the approximation as frequency increases, having certain characteristics sensitive to microscale details. We observe that the homogenized model captures the dynamic response of locally resonant media more accurately and across a wider range of frequencies than the dynamic response of media without local resonance.
\end{abstract}

Keywords: Composite, Phononic crystal, metamaterial, Local resonator, Band gap, Flexural wave propagation, Bloch-Floquet analysis, Dynamic homogenization

\section{Introduction}

The physics of systems with microstructure is governed by complex differential equations with spatially varying coefficients, leading to fields that exhibit rapid fluctuations at the microscale. Homogenization theory aims at describing such systems in terms of simpler effective or macroscopic equations, assuming these microscale variations can be averaged out (Hashin, 1983, Nemat-Nasser and Hori, 1999, Milton, 2002). In turn, the homogenized models-ordinarily developed when analyzing infinite media-are employed in investigating the physics of microstructured systems

${ }^{*}$ Corresponding author. Tel.: +1 972 778871613. E-mail address: meshmuel@technion.ac.il (G. Shmuel). 
bordered by other media. Differently from its constituents, the effective medium may exhibit extraordinary properties, in which case it is termed a metamaterial. Specifically, metamaterials in elastodynamics admit negative effective mass and stiffness, effective anisotropic mass, and capable of wave manipulation through negative refraction, filtering and steering (Milton and Willis, 2007, Bigoni et al., 2013, Celli and Gonella, 2014, Ma and Sheng, 2016, Barnwell et al., 2017).

This work is concerned with the dynamic homogenization of composite and locally resonant flexural media, whose dynamics and metamaterial properties have been extensively studied recently (e.g., Xiao et al., 2013, Carta and Brun, 2015, Chen et al., 2017, Yang et al., 2017). The Euler-Bernoulli beam model for flexural motions - the model we address in the sequel—is not only one of the fundamental models in structural engineering, it is also employed in MEMS modeling (Korvink and Paul, 2006), lattice models of materials (Ostoja-Starzewski, 2002), and constitutes a platform for the analysis of novel applications (Colquitt et al., 2014, Misseroni et al., 2016, Chen et al., 2017, Zareei et al., 2018). Different approaches were employed to describe their effective behavior; Sun et al. (2017) used an asymptotic expansion method to derive effective wave equations at low frequencies of a composite beam, rather than identifying effective properties of a homogenized medium; Antonakakis and Craster (2012) extended the exceptional high frequency homogenization theory of Craster et al. (2010) to obtain a long-scale governing equation-of a different form than the microscale equation - that is applicable at high frequencies, by perturbing about standing long-waves; Chen et al. (2017) defined frequency-dependent effective properties of a beam with periodically attached local resonators in terms of calculated macroscopic quantities; Torrent et al. (2014) developed an effective theory for inclusion-based locally resonant flexural media, based on the scattering properties of the inclusions.

The objective of the present work is twofold; (i) Develop a simple homogenization scheme that delivers the exact dispersion relation—not only at low frequencies-for composite beams and flexural systems with periodically attached local resonators; (ii) Quantify the implications of violating the fundamental homogenization assumption - that the wavelength is much larger than the microstructure —on the replacement of semi-infinite and finite systems by their homogenized models.

To achieve objective $(i)$, we examine macroscopic equations for the volume averages of the field variables. The corresponding effective coefficients are derived from micromechanical considerations and Fourier analysis, without the need to calculate the field variables at each point ( $c f$., Willis, 2009, Nemat-Nasser et al., 2011). A similar approach was applied by Nemat-Nasser and Srivastava (2011) for dynamic homogenization of laminates; as in the latter work, the local fields are actually extractable from our scheme, if wanted. Indeed, the macroscopic equations, in conjunction with the frequency-dependent effective properties, deliver the exact dispersion relation of infinite microstructured media. 
To carry out objective (ii), we investigate the reflection behavior of semi-infinite and finite periodic systems in comparison with their homogenized replacements, through a study of two scattering problems, as investigated by Srivastava and Nemat-Nasser (2014) and Joseph and Craster (2015) for laminates. In the first problem, we analyze the energy reflected from an interface between two semi-infinite media, where one is periodic and the other is its homogenized equivalent. Specifically, we explore the reflected energy dependency on the frequency and microscale details, such as the interface location within the periodic cell. In the second problem, we analyze a finite periodic beam bounded between two semi-infinite homogeneous beams, and compare its transmission spectrum with the homogenized equivalent spectrum.

The paper is organized as follows. Sec. 2 firstly revisits the problem flexural wave propagation in composite beams and systems with local resonators. Afterwards, our derivation for the macroscopic equations and effective properties is provided. In Sec. 3 we describe the semi-infinite and finite scattering problems, and derive expressions for the reflected and transmitted energy, respectively. Therein, we demonstrate that in the long-wavelength limit, our homogenized model is able to match the impedance of the original periodic system, and hence to avoid reflection. Sec. 4 studies the applicability of the homogenization model in the infinite problem and scattering problems by way of numerical examples. Sec. 5 concludes the paper, summarizing our main results and observations.

\section{Dynamic homogenization for periodic flexural systems}

\subsection{Wave propagation in periodic flexural systems}

Composite beams. Consider a beam made of alternating phases in the $x$ direction, namely, phases $a$ and $b$ of lengths $l^{(a)}$ and $l^{(b)}$, respectively. Accordingly, we have that $E(x+l)=E(x)$ and $\rho(x+l)=\rho(x)$, where $l=l^{(a)}+l^{(b)}$ is the length of the unit cell, $E(x)$ is the Young modulus and $\rho(x)$ the mass density per unit volume. The cross-section area, $A$, and the inertia moment, $I$, are uniform throughout the medium, as illustrated in Fig. 1(a).

In the absence of distributed loading, the Euler-Bernoulli beam model of flexural motion reads (see, e.g., Graff, 1975)

$$
\left[B(x) u_{, x x}\right]_{, x x}+\rho(x) A u_{, t t}=0,
$$

where $u$ is the transverse displacement and $B(x)=E(x) I$ is the bending stiffness. Assuming time 
(a)

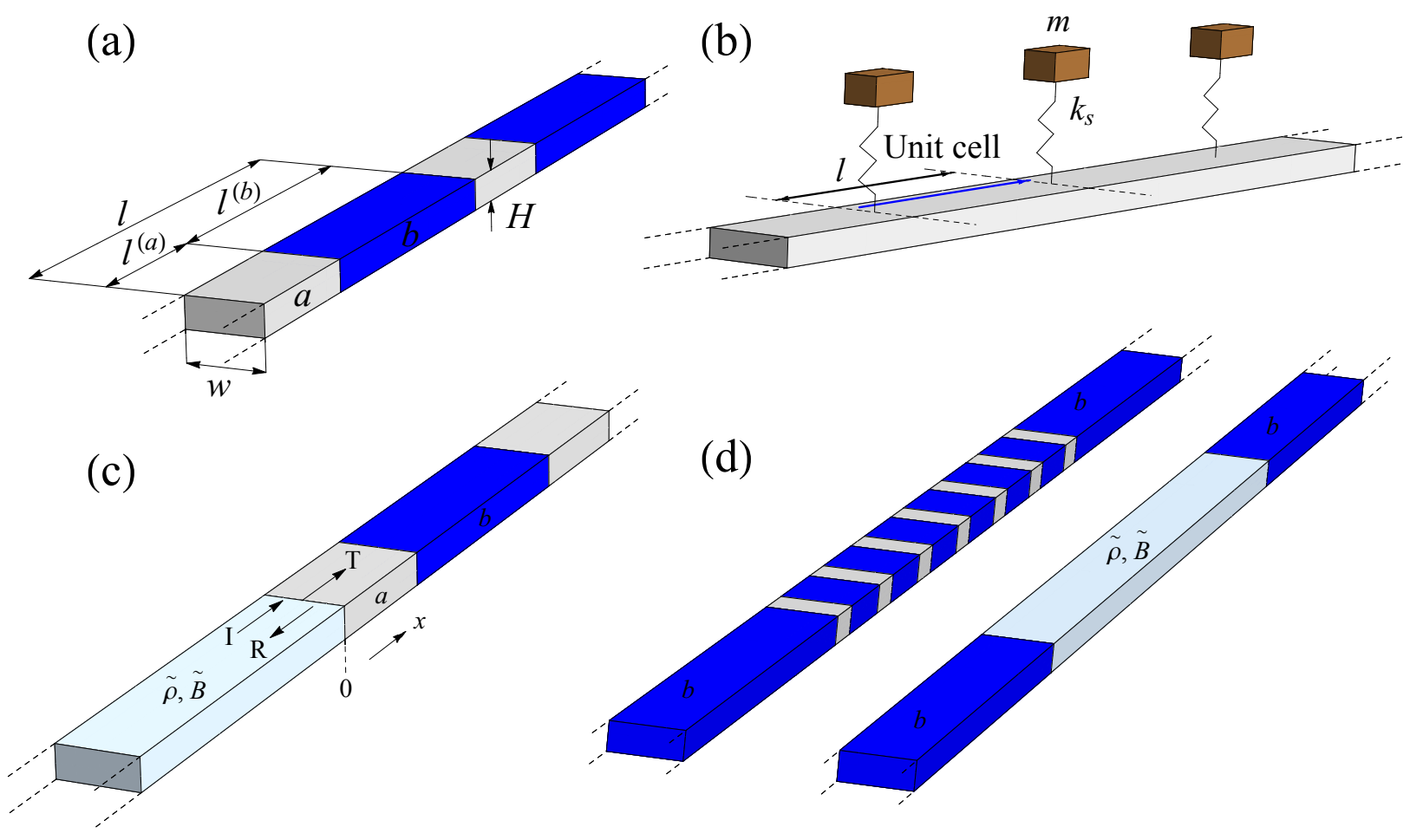

Figure 1: (a) An infinite periodic beam made of two alternating $a$ and $b$ phases. (b) A flexural system with periodically attached local resonators, modeled as mass-spring elements attached to a uniform beam. A unit cell of length $l$ comprises a resonator attached to its right end. (c) A semi-infinite periodic beam in contact at $x=0$ with its homogenized equivalent occupying the domain $x<0$. The letters $\mathrm{I}, \mathrm{R}$, and $\mathrm{T}$ denote incident, reflected and transmitted waves, respectively. (d) Two semi-infinite $b$ phases connected by a finite periodic beam (left), and connected by the homogenized beam of the same length (right).

dependency in the form $e^{-i \omega t}$, Eq. (1) can be rewritten as

$$
M_{, x x}-i \omega p=0
$$

$M=B(x) u_{, x x}$ and

$$
p=\rho(x) A u_{, t}=-\rho(x) A i \omega u
$$

is the linear momentum. For harmonic waves traveling in this periodic beam, the field variables are of the form (Bloch, 1929, Kittel, 2005)

$$
R(x, t)=R_{\mathrm{p}}(x) e^{i\left(k_{B} x-\omega t\right)},
$$

where $R$ represents the field variables, namely, $u$, and the angle of rotation, $\theta$, bending moment, 
$M$ and shear force, $V$, and $R_{\mathrm{p}}$ is periodic with the same periodicity as the unit cell; the outstanding question is to relate the Bloch wavenumber, $k_{B}$, and the frequency $\omega$. To this end, we note that in each phase, the general solution of Eq. (1) is

$$
u(x)=C^{+} e^{i k x}+D^{+} e^{-k x}+C^{-} e^{-i k x}+D^{-} e^{k x}
$$

where $k=\sqrt[4]{\rho A \omega^{2} / B}$, and the coefficients $C^{ \pm}$(resp. $D^{ \pm}$) denote the amplitudes of the propagating (resp. non-propagating) waves. It follows that the field variables at the ends $x_{0}^{(i)}$ and $x_{0}^{(i)}+l^{(i)}$ of each phase $i$ are related via ${ }^{1}$

$$
\left\{\begin{array}{c}
u\left(x_{0}^{(i)}\right) \\
\theta\left(x_{0}^{(i)}\right) k^{(a)^{-1}} \\
M\left(x_{0}^{(i)}+l^{(i)}\right) k^{(a)^{-2}} \\
V\left(x_{0}^{(i)}+l^{(i)}\right) k^{(a)^{-3}}
\end{array}\right\}=\mathrm{H}^{(i)} \cdot\left\{\begin{array}{c}
M\left(x_{0}^{(i)}\right) k^{(a)^{-2}} \\
V\left(x_{0}^{(i)}\right) k^{(a)^{-3}} \\
u\left(x_{0}^{(i)}+l^{(i)}\right) \\
\theta\left(x_{0}^{(i)}+l^{(i)}\right) k^{(a)^{-1}}
\end{array}\right\}
$$

where the $k^{(a)}$ is the value that $k$ takes in phase $a$, and $\mathrm{H}^{(i)}$ is given in Appendix A. Using the continuity of the field variables and Eq. (4), the dispersion relation $k_{B}(\omega)$ of a periodic beam can be determined by solving the generalized eigenproblem (Tan, 2010)

$$
\left[\begin{array}{cc}
-\mathrm{I}_{2} & \mathrm{H}_{11} \\
0_{2} & \mathrm{H}_{21}
\end{array}\right] \cdot \mathrm{s}_{\mathrm{m}}(x)=e^{i k_{B} l}\left[\begin{array}{cc}
-\mathrm{H}_{12} & 0_{2} \\
-\mathrm{H}_{22} & \mathrm{I}_{2}
\end{array}\right] \cdot \mathrm{s}_{\mathrm{m}}(x)
$$

here $\mathrm{H}_{11}, \mathrm{H}_{12}, \mathrm{H}_{21}, \mathrm{H}_{22}$ denote the $2 \times 2$ sub-blocks of the hybrid matrix corresponding to the unit cell whose ends are at $x$ and $x+l$, and $\mathrm{s}_{\mathrm{m}}(x)=\left\{u(x), \theta(x) k^{(a)^{-1}}, M(x) k^{(a)^{-2}}, V(x) k^{(a)^{-3}}\right\}^{\top}$ is the modified state vector. The characteristic polynomial associated with Eq. (7) provides

$$
\cos k_{B} l=\frac{-a_{2} \pm \sqrt{a_{2}^{2}-4 a_{1}\left(a_{3}-2 a_{1}\right)}}{4 a_{1}}
$$

where $a_{1}=\operatorname{det} \mathrm{H}_{12}$ and the coefficients $a_{2}$ and $a_{3}$ are cumbersome functions of the matrix elements in Eq. (7), omitted for brevity.

Note that if $k_{B} l$ is a solution, then so are $2 \pi n \pm k_{B} l$, for $n \in \mathbb{Z}$. The region $-\pi \leqslant k_{B} l \leqslant \pi$ is called the $1^{\text {st }}$ Brillouin zone. The $2^{\text {nd }}$ Brillouin zone comprises the negative region $-2 \pi+k_{B} l$ and the positive region $2 \pi-k_{B} l$, and so forth. To determine the range of propagating and attenuating

\footnotetext{
${ }^{1}$ The standard transfer matrix formulation is given, e.g., in Carta and Brun (2015) and Xu et al. (2016).
} 
frequencies it is sufficient to examine the irreducible $1^{\text {st }}$ Brillouin zone, $0 \leqslant k_{B} l \leqslant \pi$ (Farzbod and Leamy, 2011); if $k_{B}$ is complex, then the frequency belongs to a gap, i.e., there is no propagating solution, and waves at this frequency decay.

Locally resonant flexural systems. The dispersion relation derivation of flexural systems with periodically attached local resonators is summarized next (Yu et al., 2006, Xiao et al., 2013, Shuguang et al., 2015, Carta and Brun, 2015). Such systems are analyzed as uniform EulerBernoulli beams connected periodically to harmonic oscillators modeled as mass $(m)$-spring $\left(k_{s}\right)$ elements, as shown in Fig. 1(b). The equation of motion for this model can be expressed as

$$
\left[B(x) u_{, x x}\right]_{, x x}-\rho(x) \omega^{2} A u(x)=-\sum_{n} \frac{k_{s} \omega^{2} u\left(x_{n}\right)}{\omega^{2}-\omega_{0}^{2}} \delta\left(x-x_{n}\right),
$$

where $\delta(x)$ is the Dirac delta function, $\omega_{0}=\sqrt{\frac{k_{s}}{m}}$ and the factor $-\frac{k_{s} \omega^{2} u\left(x_{n}\right)}{\left(\omega^{2}-\omega_{0}^{2}\right)}$ is the force acting on the beam at the connection points $x_{n}=n l, n \in \mathbb{Z}$. Note that $\sum_{n} u\left(x_{n}\right) \delta\left(x-x_{n}\right)=u(x) \sum_{n} \delta\left(x-x_{n}\right)$, hence we can recast Eq. (9) in a form that is similar to Eq. (1), by replacing $\rho(x)$ with $\hat{\rho}(x)=$ $\rho(x)-\frac{k_{s}}{\left(\omega^{2}-\omega_{0}^{2}\right) A} \sum_{n} \delta\left(x-x_{n}\right)$.

As in the previous derivation, a hybrid matrix and Bloch-Floquet analysis is carried out by considering a unit cell of length $l$ with a resonator attached to its right end. The resultant hybrid matrix is equal to the hybrid matrix of a homogeneous cell plus the term $\frac{k_{S} \omega^{2}}{\left(\omega^{2}-\omega_{0}^{2}\right)}$ at its $(4,3)$ entry (Appendix A). The appended term comes from the change in the value of the shear force at the connection point. The corresponding dispersion relation is Eq. (8) with modified $a_{i}$ according to the new hybrid matrix.

\subsection{Effective properties}

Our derivation of the effective properties for flexural systems relies on volume averages of field variables, similarly to the procedure developed in Nemat-Nasser et al. (2011) for laminates (see also Willis, 2009). By construction, the resultant homogenized formulation satisfies macroscopic field equations, and recovers exactly the dispersion relation of composite and locally resonant beams.

To derive macroscopic relations in terms of mean quantities, we multiply Eq. (2) by $e^{-i k_{B} X}$ and obtain

$$
\left[M_{\mathrm{p}}(x) e^{i k_{B}(x-X)}\right], x x-i \omega p_{\mathrm{p}}(x) e^{i k_{B}(x-X)}=0 .
$$


In terms of $\chi=x-X$, Eq. (10) reads

$$
\left[M_{\mathrm{p}}(X+\chi) e^{i k_{B} \chi}\right], \chi \chi-i \omega p_{\mathrm{p}}(X+\chi) e^{i k_{B} \chi}=0 \text {. }
$$

Eq. (11) with respect to $X$ over the unit cell provides

$$
\left[\left\langle M_{\mathrm{p}}\right\rangle^{i k_{B} \chi}\right], \chi \chi-i \omega\left\langle p_{\mathrm{p}}\right\rangle e^{i k_{B} \chi}=0
$$

where the average of each one of the periodic parts is defined as

$$
\langle\circ\rangle=\frac{1}{l} \int_{-l / 2}^{l / 2}(0) \mathrm{d} X \text {. }
$$

The averaged field variables are given by

$$
\bar{\zeta}(x)=\left\langle\varsigma_{\mathrm{p}}\right\rangle e^{i k_{B} x}, \varsigma=M, p, u
$$

and satisfy a governing equation similar to Eq. (2), in the form

$$
\bar{M}(x)_{, x x}-i \omega \bar{p}(x)=0 .
$$

We complete our formulation with the following macroscopic counterparts of the moment-displacement relation and Eq. (3)

$$
\bar{M}(x)=\tilde{B} \bar{u}_{, x x}(x), \quad \bar{p}(x)=-i \omega A \tilde{\rho} \bar{u}(x)
$$

which define the effective bending stiffness, $\tilde{B}$, and effective mass density, $\tilde{\rho}$. Eq. (16) yields with Eq. (15) a frequency-wavenumber relation whose form is analogous to that of a homogeneous beam, namely,

$$
k_{B}^{4}-\omega^{2} \frac{A \tilde{\rho}}{\tilde{B}}=0
$$

Eq. (17) reproduces precisely the dispersion relation of flexural composite and locally resonant systems, through the frequency-dependent effective properties $\tilde{\rho}$ and $\tilde{B}$ given in Eq. (15), as will be demonstrated in the sequel. 


\subsection{Analytic formulas for calculating the effective properties}

The common procedure to determine the effective properties requires the calculation of the local fields and integration of their periodic parts over the unit cell. Here we employ a different approach, incorporating Fourier analysis. Firstly, we consider the Bloch form of $u, M$ and $p$, and expand their periodic part into Fourier series, as well as the quantities $B$ and $\rho$ (or $\hat{\rho}$ for the locally resonant beam). Accordingly, we have that

$$
\varsigma(x)=e^{i k_{B} x} \sum_{m} \varsigma_{\mathrm{F}}(m) e^{\frac{2 i \pi m x}{l}}, \quad \varsigma=u, M, p, \quad m \in \mathbb{Z},
$$

where

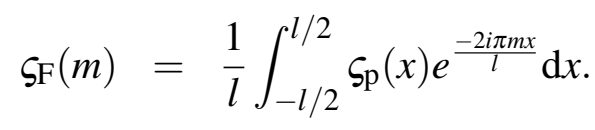

In Eq. (18), the term with $m=0$ describes the part of $\varsigma(x)$ that varies slower with $x$ than the part associated with the rest of the terms. It follows that

$$
\bar{\varsigma}(x)=\varsigma_{\mathrm{F}}(m=0) e^{i k_{B} x}=\left\langle\varsigma_{\mathrm{p}}\right\rangle e^{i k_{B} x}
$$

Substitution of the Fourier expansions into the relation $M=B(x) u_{, x x}$ and Eq. (3) delivers a relation between $\left\langle M_{\mathrm{p}}\right\rangle$ and $\left\langle u_{\mathrm{p}}\right\rangle$, and between $\left\langle p_{\mathrm{p}}\right\rangle$ and $\left\langle u_{\mathrm{p}}\right\rangle$, namely,

$$
\begin{aligned}
& \left\langle M_{\mathrm{p}}\right\rangle=-B_{\mathrm{F}}(0) k_{B}^{2}\left\langle u_{\mathrm{p}}\right\rangle-\sum_{m \neq 0} B_{\mathrm{F}}(-m)\left(k_{B}+\frac{2 \pi m}{l}\right)^{2} u_{\mathrm{F}}(m), \\
& \left\langle p_{\mathrm{p}}\right\rangle=-\rho_{\mathrm{F}}(0) i \omega A\left\langle u_{\mathrm{p}}\right\rangle-i \omega A \sum_{m \neq 0} \rho_{\mathrm{F}}(-m) u_{\mathrm{F}}(m) .
\end{aligned}
$$

We clarify that in Eq. (22) and the sequel, the terms $\rho_{\mathrm{F}}$ are replaced by $\hat{\rho}_{\mathrm{F}}$ when the locally resonant beam is addressed. We eliminate $u_{\mathrm{F}}(m)$ from Eqs. (21-22) by expressing it in terms of $\left\langle u_{\mathrm{p}}\right\rangle$, similarly to Cerdán-Ramírez et al. (2009), who carried out a related analysis for photonic crystals. In our case, this relation is obtained from the equation describing the propagation of flexural waves in beams, i.e., Eq. (1). Substituting into this equation the Fourier expansions provides

$$
\sum_{m^{\prime}} \mathrm{Q}\left(k_{B} ; m, m^{\prime}\right) u_{\mathrm{F}}\left(m^{\prime}\right)=0
$$


where

$$
\mathrm{Q}\left(k_{B} ; m, m^{\prime}\right)=\left(k_{B}+\frac{2 \pi m}{l}\right)^{2} B_{\mathrm{F}}\left(m-m^{\prime}\right)\left(k_{B}+\frac{2 \pi m^{\prime}}{l}\right)^{2}-\omega^{2} A \rho_{\mathrm{F}}\left(m-m^{\prime}\right) .
$$

We write the coefficients $u_{\mathrm{F}}(m \neq 0)$ in terms of $u_{\mathrm{F}}(m=0)=\left\langle u_{\mathrm{p}}\right\rangle$ via the equations for $m \neq 0$ in Eq. (23), and obtain

$$
u_{\mathrm{F}}(m \neq 0)=-\sum_{m^{\prime} \neq 0} \mathrm{Q}_{s}^{-1}\left(k_{B} ; m, m^{\prime}\right) \mathrm{Q}\left(k_{B} ; m^{\prime}, 0\right)\left\langle u_{\mathrm{p}}\right\rangle .
$$

Here, $Q_{s}\left(k_{B} ; m, m^{\prime}\right)$ is a sub-matrix, obtained from the matrix represented in Eq. (24) after eliminating its row (resp. column) for $m=0$ (resp. $m^{\prime}=0$ ). The effective properties $\tilde{B}$ and $\tilde{\rho}$ are determined by substituting Eq. (25) into Eqs. (21-22) and utilizing the macroscopic relations $\left\langle M_{\mathrm{p}}\right\rangle=-\tilde{B} k_{B}^{2}\left\langle u_{\mathrm{p}}\right\rangle$ and $\left\langle p_{\mathrm{p}}\right\rangle=-i \omega A \tilde{\rho}\left\langle u_{\mathrm{p}}\right\rangle$. The end result reads

$$
\begin{aligned}
& \tilde{B}\left(k_{B}, \omega\right)=B_{\mathrm{F}}(0)+\sum_{m \neq 0} B_{\mathrm{F}}(-m)\left(k_{B}+\frac{2 \pi m}{l}\right)^{2} w_{\mathrm{F}}(m), \\
& w_{\mathrm{F}}(m \neq 0)=-\sum_{m^{\prime} \neq 0} \mathrm{Q}_{s}^{-1}\left(k_{B} ; m, m^{\prime}\right)\left[\left(k_{B}+\frac{2 \pi m^{\prime}}{l}\right)^{2} B_{\mathrm{F}}\left(m^{\prime}\right)-\left(\frac{\omega}{k_{B}}\right)^{2} A \rho_{\mathrm{F}}\left(m^{\prime}\right)\right] . \\
& \tilde{\rho}\left(k_{B}, \omega\right)=\rho_{\mathrm{F}}(0)+\sum_{m \neq 0} \rho_{\mathrm{F}}(-m) v_{\mathrm{F}}(m), \\
& v_{\mathrm{F}}(m \neq 0)=-\sum_{m^{\prime} \neq 0} \mathrm{Q}_{s}^{-1}\left(k_{B} ; m, m^{\prime}\right)\left[\left(k_{B}+\frac{2 \pi m^{\prime}}{l}\right)^{2} B_{\mathrm{F}}\left(m^{\prime}\right) k_{B}^{2}-\omega^{2} A \rho_{\mathrm{F}}\left(m^{\prime}\right)\right] .
\end{aligned}
$$

We remark that for the locally resonant beam, the components $\hat{\rho}_{\mathrm{F}}\left(m-m^{\prime}\right)$ are negative at $1<\frac{\omega}{\omega_{0}}<\sqrt{1+\frac{m}{\rho A l}}$. Notably, the effective properties $\tilde{B}$ and $\tilde{\rho}$ depend on the wavenumber $k_{B}$ and the frequency $\omega$. Eq. (23) provides the dispersion relation $k_{B}(\omega)$ of the flexural system, through its implied condition

$$
\operatorname{det} Q=0 .
$$

(Of course, this calculation of the dispersion relation is not needed, having Eq. (8) at hand; we provide it to argue that our approach can be generalized to cases in which exact dispersion relations are not accessible.) In turn, the frequency-dependent effective properties $\tilde{B}$ and $\tilde{\rho}$ are obtained by substituting $\left(k_{B}, \omega\right)$ pairs into Eqs. (26-27). Alternatively, the dispersion relation and the effective properties can be evaluated using the following iterative procedure. Firstly, the static weighted 
averages

$$
\tilde{B}_{0}=l\left(\frac{l^{(a)}}{B^{(a)}}+\frac{l^{(b)}}{B^{(b)}}\right)^{-1}, \tilde{\rho}_{0}=\frac{l^{(a)} \rho^{(a)}+l^{(b)} \rho^{(b)}}{l}
$$

are substituted into Eq. (17) as $\tilde{B}$ and $\tilde{\rho}$ to obtain an initial dispersion relation $\left(k_{B}, \omega\right)$; the latter is substituted into Eqs. (26-27) to find a first iteration of the frequency-dependent properties $\tilde{B}\left(k_{B}, \omega\right)$ and $\tilde{\rho}\left(k_{B}, \omega\right)$, which are substituted back to Eq. (17) to evaluate the next iteration of the dispersion relation, and so forth, until convergence.

We emphasize that our scheme evades the need to calculate the microscopic displacement field; it is actually extractable from our scheme, by substituting back Q into Eq. (23) to calculate $u_{\mathrm{F}}(m)$, and in turn, $u(x)$.

\section{Reflection and transmission in interface problems}

As discussed by Srivastava and Nemat-Nasser (2014) and Joseph and Craster (2015), to justify a replacement of the periodic medium by fictitious homogeneous medium with effective properties, their response to interface problems should be similar. Srivastava and Nemat-Nasser (2014) suggested to quantify this similarity by the reflected energy (see also Herzig Sheinfux et al., 2014, Joseph and Craster, 2015, Amirkhizi, 2017). Accordingly, we analyze next the reflection and transmission at the interface of semi-infinite and finite periodic flexural systems, in comparison with their dynamic homogenized equivalents, as illustrated in Figs. 1(c) and 1(d).

\subsection{Two contiguous semi-infinite beams}

We consider a semi-infinite fictitious homogeneous beam with bending stiffness $\tilde{B}$ and mass density $\tilde{\rho}$ occupying the domain $x<0$. The beam is perfectly bounded at $x=0$ to a semi-infinite periodic system, whose properties are described in Sec. 2.1, occupying the domain $x>0$, see Fig. 1(c). The fictitious beam serves as the homogenized equivalent of the periodic system, hence its properties satisfy the dispersion relation (17), with a wavenumber that coincides with the Bloch wavenumber $k_{B}$ of waves in the periodic system. At the interface, a positive-going wave $C^{+} e^{i k_{B} x}$ excited from the left is partially reflected in the form of a negative-going wave $C^{-} e^{-i k_{B} x}$ and attenuating wave $D^{-} e^{k_{B} x}$. A transmitted wave in the semi-infinite periodic domain is created too, comprising positive-going wave $C_{\mathrm{p}}^{+} u_{\mathrm{p}}(x) e^{i k_{B} x}$ and attenuating wave $D^{+} e^{-k_{B} x}$. The attenuating waves-required for the field variables to be continuous across the interface-are negligible far from the interface. 
From the corresponding continuity conditions at the interface we obtain

$$
\begin{aligned}
& C^{+}+C^{-}+D^{-}=C_{\mathrm{p}}^{+} u(0)+D^{+}, \\
& i C^{+}-i C^{-}+D^{-}=C_{\mathrm{p}}^{+} \frac{\theta(0)}{k_{B}}-D^{+}, \\
& -\tilde{B} C^{+}-\tilde{B} C^{-}+\tilde{B} D^{-}=C_{\mathrm{p}}^{+} \frac{M(0)}{k_{B}^{2}}+\tilde{B} D^{+}, \\
& i \tilde{B} C^{+}-i \tilde{B} C^{-}-\tilde{B} D^{-}=C_{\mathrm{p}}^{+} \frac{V(0)}{k_{B}^{3}}+\tilde{B} D^{+},
\end{aligned}
$$

where $u(0), \theta(0), M(0)$ and $V(0)$ are the solutions of the hybrid matrix generalized eigenproblem (7) at $x=0$. Note that the attenuating wave $D^{+} e^{-k_{B} x}$ was treated as a wave solution corresponding to a homogeneous medium having the dynamic effective properties $\tilde{B}$ and $\tilde{\rho}$. The continuity conditions (30) constitute a system of linear equations, from which we obtain the reflection coefficient $r \equiv \frac{C^{-}}{C^{+}}$, namely,

$$
r=\frac{i k_{B}^{3} \tilde{B} u(0)-i k_{B} M(0)-\left[V(0)+k_{B}^{2} \tilde{B} \theta(0)\right]}{i k_{B}^{3} \tilde{B} u(0)-i k_{B} M(0)+\left[V(0)+k_{B}^{2} \tilde{B} \theta(0)\right]}
$$

or

$$
r=\frac{\gamma-1}{\gamma+1}
$$

where

$$
\gamma=\frac{i k_{B}^{3} \tilde{B} u(0)-i k_{B} M(0)}{V(0)+k_{B}^{2} \tilde{B} \theta(0)}
$$

The normalized reflected energy equals $|r|^{2}$, and vanishes when $\gamma=1$. Following Srivastava and Nemat-Nasser (2014), we use it to quantify the applicability of the homogenized models, in our context of flexural systems. Furthermore, bearing in mind that both the reflective energy and the effective properties depend on the chosen solution of $k_{B}$, Srivastava and Nemat-Nasser (2014) suggested to use energy conservation requirements to determine which solution should be used for $r$, and, in turn, calculating $\tilde{\rho}$ and $\tilde{B}$. This is demonstrated in the sequel.

Long-wavelength limit and reflection dependency on the interface location. It is expected that in the long-wavelength limit $\gamma \approx 1$ and therefore $|r|^{2} \approx 0$. We now verify that our derivation for the effective properties meets this expectation. Since $e^{i k_{B} x}=1$ at $x=0^{+}$, we have that

$$
u(0)=u_{\mathrm{p}}(0), \theta(0)=\theta_{\mathrm{p}}(0), M(0)=M_{\mathrm{p}}(0), V(0)=V_{\mathrm{p}}(0)
$$


In the long-wavelength limit, the periodic part of the displacement varies slowly over the unit cell, its derivatives practically vanish and we have $\theta_{\mathrm{p}}(0) \approx i k_{B} u_{\mathrm{p}}(0), V_{\mathrm{p}}(0) \approx-i k_{B} M_{\mathrm{p}}(0)$. Then, the parameter $\gamma$ becomes

$$
\gamma \approx \frac{i k_{B}^{3} \tilde{B} u_{\mathrm{p}}(0)-i k_{B} M_{\mathrm{p}}(0)}{-i k_{B} M_{\mathrm{p}}(0)+k_{B}^{2} \tilde{B} i k_{B} u_{\mathrm{p}}(0)}=1
$$

We have that $\gamma \approx 1$ and, in turn, $r \approx 0$ as it should. Note that this result is only satisfied for a homogeneous beam having the effective properties given in Eq. (16). If this medium is replaced by a homogeneous one having mass density $\rho_{h} \neq \tilde{\rho}$ and bending stiffness $B_{h} \neq \tilde{B}$ (which also satisfy the dispersion relation of the periodic beam), then Eqs. (30) will not yield vanishing $r$ in the low frequency limit. Stated differently, in the long-wavelength limit the periodic structure effectively behaves as a homogeneous beam whose bending stiffness is $\tilde{B} \approx-M_{\mathrm{p}}(0) / k_{B}^{2} u_{\mathrm{p}}(0)$.

Note that the result in Eq. (35) is independent of the interface location within the unit cell. Generally, however, the reflected energy depends on that location, and hence, so does the applicability of the homogenized model. This is due to the dependency of the field variables on the position, and in turn, the parameter $\gamma$, as we numerically demonstrate in Sec. 4.

\subsection{Finite system bounded by two semi-infinite homogeneous media}

We analyze the transmission through a periodic system bounded by two homogeneous semi-infinite media, see Fig. 1(d). Specifically, we consider an incident wave of amplitude $C^{+}(L)$ from the left, and calculate the normalized transmitted energy, $|t|^{2}=\left|\frac{C^{+}(R)}{C^{+}(L)}\right|^{2}$, where the $C^{+}(R)$ is the magnitude of the transmitted wave to the right. To evaluate $|t|^{2}$, we derived an expression using the hybrid matrix of the intermediate finite system, $\mathrm{H}_{\mathrm{f}}$, determined according to the procedure detailed in Appendix A. For simplicity, we assume that the semi-infinite media are made of phase $b$. The corresponding components of the modified state vector $\mathrm{s}_{\mathrm{m}}(x)$ are

$$
\begin{aligned}
& \left\{\begin{array}{c}
u(x) \\
\theta(x) k^{(a)^{-1}}
\end{array}\right\}=\mathrm{Q}_{u \theta}^{+}(x) \cdot \mathrm{c}^{+}+\mathrm{Q}_{u \theta}^{-}(x) \cdot \mathrm{c}^{-}, \\
& \left\{\begin{array}{c}
M(x) k^{(a)^{-2}} \\
V(x) k^{(a)^{-3}}
\end{array}\right\}=\mathrm{Q}_{M V}^{+}(x) \cdot \mathrm{c}^{+}+\mathrm{Q}_{M V}^{-}(x) \cdot \mathrm{c}^{-},
\end{aligned}
$$


where

$$
\begin{aligned}
& \mathrm{c}^{ \pm}=\left\{\begin{array}{c}
C^{ \pm} \\
D^{ \pm}
\end{array}\right\}, \mathrm{Q}_{u \theta}^{ \pm}(x)=\left[\begin{array}{cc}
e^{ \pm i k^{(b)} x} & e^{\mp k^{(b)} x} \\
\pm i \frac{k^{(b)}}{k^{(a)}} e^{ \pm i k^{(b)} x} & \mp \frac{k^{(b)}}{k^{(a)}} e^{\mp k^{(b)} x}
\end{array}\right], \\
& \mathrm{Q}_{M V}^{ \pm}(x)=B^{(b)}\left(\frac{k^{(b)}}{k^{(a)}}\right)^{2}\left[\begin{array}{cc}
-e^{ \pm i k^{(b)} x} & e^{\mp k^{(b)} x} \\
\pm i \frac{k^{(b)}}{k^{(a)}} e^{ \pm i k^{(b)} x} & \pm \frac{k^{(b)}}{k^{(a)}} e^{\mp k^{(b)} x}
\end{array}\right] .
\end{aligned}
$$

Here, $B^{(b)}$ and $k^{(b)}$ denote, respectively, the bending stiffness and the wavenumber in phase $b$. The scattering matrix $S$

$$
\begin{aligned}
\mathrm{S}= & {\left[\left[\begin{array}{cc}
\mathrm{Q}_{u \theta}^{-}(0) & 0_{2} \\
0_{2} & \mathrm{Q}_{M V}^{+}(0)
\end{array}\right]-\mathrm{H}_{\mathrm{f}} \cdot\left[\begin{array}{cc}
\mathrm{Q}_{M V}^{-}(0) & 0_{2} \\
0_{2} & \mathrm{Q}_{u \theta}^{+}(0)
\end{array}\right]\right]^{-1} } \\
& \cdot\left[\mathrm{H}_{\mathrm{f}} \cdot\left[\begin{array}{cc}
\mathrm{Q}_{M V}^{+}(0) & 0_{2} \\
0_{2} & \mathrm{Q}_{u \theta}^{-}(0)
\end{array}\right]-\left[\begin{array}{cc}
\mathrm{Q}_{u \theta}^{+}(0) & 0_{2} \\
0_{2} & \mathrm{Q}_{M V}^{-}(0)
\end{array}\right]\right]
\end{aligned}
$$

$\left(\mathrm{O}_{2}\right.$ is the $2 \times 2$ null matrix) relates the amplitudes at the semi-infinite media, namely,

$$
\left\{\begin{array}{l}
c^{-}(\mathrm{L}) \\
\mathrm{c}^{+}(\mathrm{R})
\end{array}\right\}=\mathrm{S} \cdot\left\{\begin{array}{l}
\mathrm{c}^{+}(\mathrm{L}) \\
\mathrm{c}^{-}(\mathrm{R})
\end{array}\right\} .
$$

In terms of $S$, the normalized transmitted energy is

$$
|t|^{2}=\mid\left\{\left.\begin{array}{ll}
1 & 0
\end{array} \cdot \mathrm{S}_{21} \cdot\left\{\begin{array}{l}
1 \\
0
\end{array}\right\}\right|^{2},\right.
$$

where $S_{21}$ is the $2 \times 2$ bottom-left block of S. Expression (40) is also used to evaluate the normalized transmitted energy when the intermediate finite system is a locally resonant beam. In this case, the matrix $\mathrm{H}_{\mathrm{f}}$ is based on the modified hybrid matrix, described in Sec. 2.1.

\section{Numerical calculations}

We quantify next the applicability of our homogenization scheme and study its dependency on the wavelength through numerical realization of the previous derivations. This is carried out firstly for composite media homogenization, and subsequently for locally resonant media. 


\begin{tabular}{cccc}
\hline Phase & $\rho\left(\mathrm{kg} / \mathrm{m}^{3}\right)$ & $E(\mathrm{~Pa})$ & length $(\mathrm{m})$ \\
\hline$a$ & 1000 & $2 \times 10^{9}$ & 0.03 \\
$b$ & 3000 & $200 \times 10^{9}$ & 0.04 \\
\hline
\end{tabular}

Table 1: Geometrical and physical properties of the phases comprising the periodic beam. The width and thickness of the beam are $0.02 \mathrm{~m}$ and $0.0016 \mathrm{~m}$, respectively.

\subsection{Homogenization of composite beams}

Dispersion relation and effective parameters. Consider an exemplary periodic beam, whose phase geometrical and physical properties are given in Tab. 1. The first four bands, calculated by the exact relation (8), are given by the continuous blue curves in Fig. 2(a). The imaginary part of $k_{B}$, associated with the gaps, is depicted by the continuous red curve.

Figs. 2(b) and 2(c) show the frequency-dependent effective properties $\tilde{\rho}$ and $\tilde{B}$, respectively, calculated using our scheme, i.e., via Eqs. (26-27), when truncating the Fourier series at $m=200$. Across the first band, the effective properties are evaluated using $k_{B}$ solutions in the $1^{\text {st }}$ Brillouin zone, while solutions in the $2^{\text {nd }}$ (resp. $3^{\text {rd }}$ and $4^{\text {th }}$ ) Brillouin zone are used across the second (resp. third and fourth) band range. We properly obtain real values across the frequencies of the bands, and complex values across the frequencies of the gaps. The homogenized dispersion relation (17) is evaluated with these calculated effective properties in Fig. 2(a), illustrated by the circle (real part) and diamond (imaginary part) marks. Indeed, the exact and homogenized dispersion relations are in excellent agreement. Notably, this agreement extends beyond the fundamental Bloch band at low frequencies.

As mentioned, if $\left(\omega, k_{B} l\right)$ satisfy the exact dispersion relation, then so are $\left(\omega, 2 \pi n \pm k_{B} l\right)$ for integer $n$. Willis (2013) questioned if this ambiguity in the value of $k_{B}$ extends to homogenized models; we find that for the homogenized dispersion relation with $\tilde{\rho}$ and $\tilde{B}$, only a unique choice of $k_{B}$ solution recovers the exact dispersion relation. This is demonstrated in panels (d-f), where $k_{B}$ values in the $1^{\text {st }}$ Brillouin zone were chosen when calculating $\tilde{\rho}, \tilde{B}$, and the dispersion relation across the second band. Contrary to panels (a-c), were the choice of the $2^{\text {nd }}$ Brillouin zone recovers the second band, here the homogenized dispersion relation diverges from it. Similar divergence occurs at higher bands where $k_{B}$ solutions other than those employed in panel (d-f) are used; for brevity, this illustration is omitted.

According to our formulation, the dynamic effective properties are determined from Fourier coefficients $\rho(m), B(m)$ which, for a fixed unit cell length $l$, are independent of how the unit cell of the periodic system is represented. Consequently, the curves reported in Figs. 2(b) and 2(c) are also independent of the unit cell representation. 

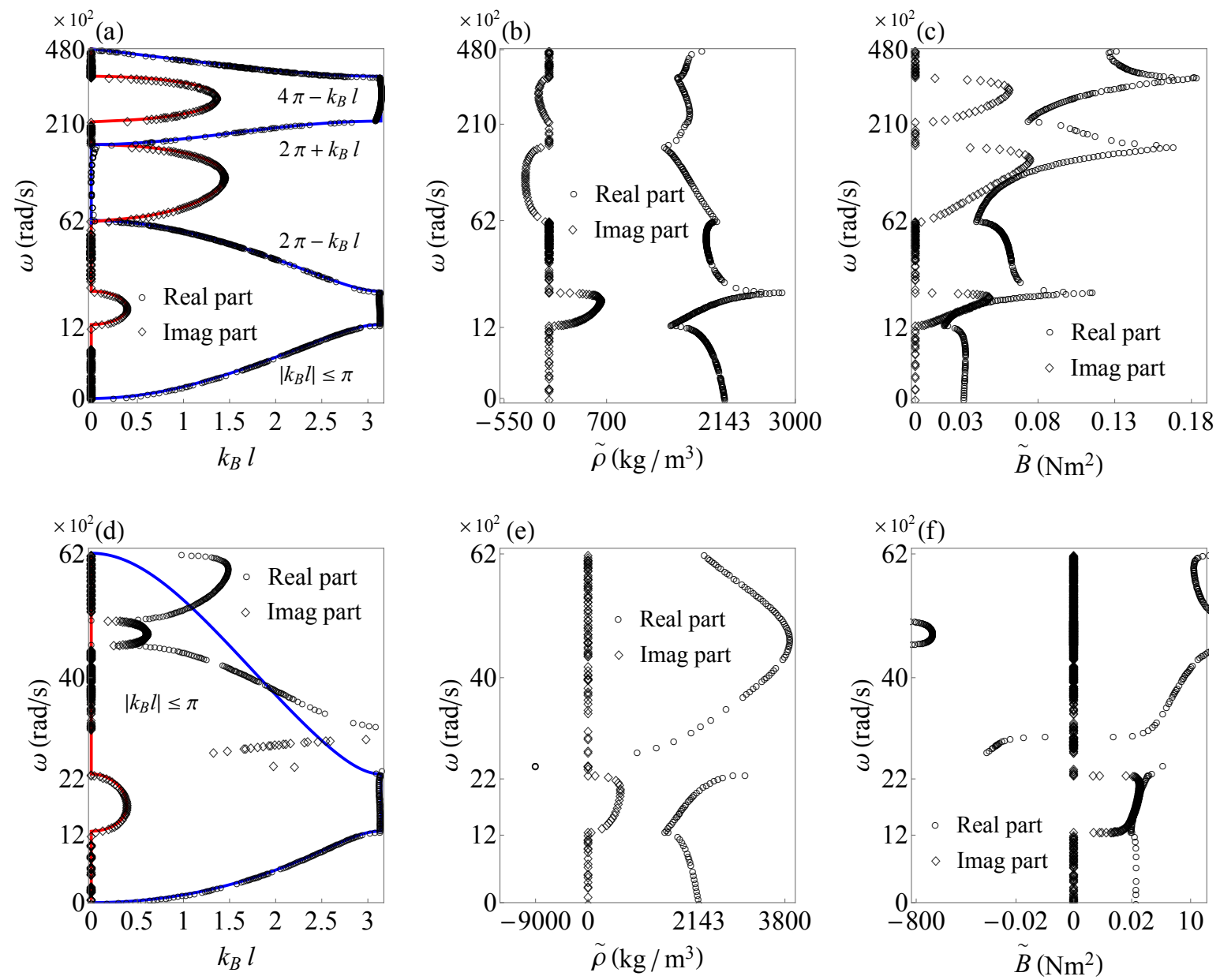

Figure 2: (a) Band diagram of the exemplary composite, truncated at the fifth band. The blue (resp. red) curve corresponds to the real (resp. imaginary) part evaluated from the exact relation (8). Circle (resp. diamond) marks correspond to the real (resp. imaginary) part evaluated from the homogenized dispersion relation (17). (b) Effective dynamic mass density $\tilde{\rho}$ and (c) effective dynamic bending stiffness $\tilde{B}$ employed in panel (a), calculated using increasing values of $k_{B}$. Panels (d-f) are the counterparts of (a-c), respectively, when the band diagram is truncated at the second gap, and $k_{B}$ is restricted to the $1^{\text {st }}$ Brillouin zone.

Contiguous semi-infinite beams. We continue to the interface problem between the semi-infinite composite and its homogenized equivalent. In Sec. 3 we showed that the spatial low variation of the displacement and bending moment fields at low frequencies implies that $r \approx 0$, and hence the homogenized model captures the reflectance behavior of the periodic beam. As the frequency is increased, the fields $u_{\mathrm{p}}(x)$ and $M_{\mathrm{p}}(x)$ fluctuate more rapidly, reflection becomes significant, and the suitability of the homogenized model deteriorates. We demonstrate this in Fig. 3, by plotting the normalized periodic part of the displacement field (panel a), $\frac{\left|u_{\mathrm{p}}(x)\right|}{\left\langle\left|u_{\mathrm{p}}(x)\right|\right\rangle}$, and the bending moment (panel b), $\frac{\left|M_{\mathrm{p}}(x)\right|}{\left\langle\left|M_{\mathrm{p}}(x)\right|\right\rangle}$, over the unit cell, at the frequencies $10 \frac{\mathrm{rad}}{\mathrm{s}}$ (solid curves), $100 \frac{\mathrm{rad}}{\mathrm{s}}$ (dotted 

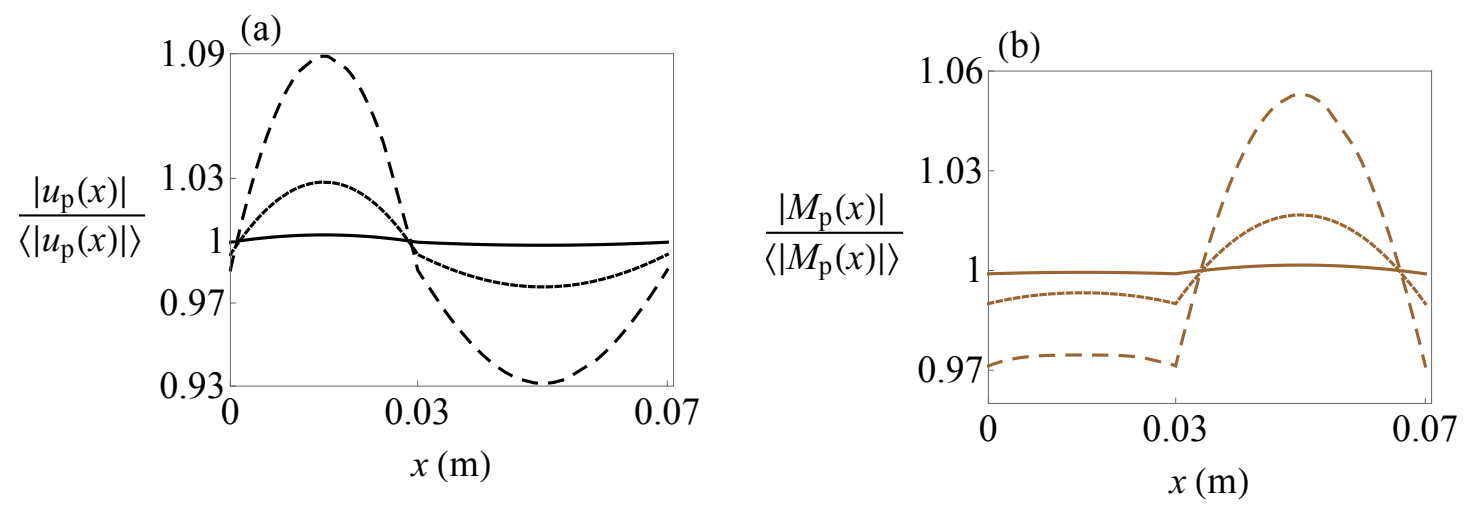

Figure 3: The fields $\frac{\left|u_{\mathrm{p}}(x)\right|}{\left\langle\left|u_{\mathrm{p}}(x)\right|\right\rangle}$ (black curves), and $\frac{\left|M_{\mathrm{p}}(x)\right|}{\left\langle\left|M_{\mathrm{p}}(x)\right|\right\rangle}$ (brown curves) over the unit cell at 10, 100 and $300 \frac{\mathrm{rad}}{\mathrm{s}}$ (solid, dotted and dashed curves, respectively).

curves), and $300 \frac{\mathrm{rad}}{\mathrm{s}}$ (dashed curves). It can be seen that the periodic parts of the displacement and the bending moment are practically constants for frequencies below $100 \frac{\mathrm{rad}}{\mathrm{s}}$, while having a more significant variation at $300 \frac{\mathrm{rad}}{\mathrm{s}}$. At this frequency, the normalized periodic part of the displacement reach a maximum deviation of 0.09 from unity. In accordance with the increase in mode fluctuation at higher frequencies, reflectance in the semi-infinite interface problem increases too; for instance, when considering an interface at the middle of the phase $a$, the reflection at the frequencies 10, 100 and $300 \frac{\mathrm{rad}}{\mathrm{s}}$ is $6.32 \times 10^{-7}, 7.04 \times 10^{-5}$, and $7.84 \times 10^{-4}$, respectively.

The dependency of the reflected energy on the wavelength and interface location is notably demonstrated in Fig. 4(a) by plotting $\omega-|r|^{2}$ diagram for four different interface locations, illustrated in the inset. Indeed, we observe that the reflected energy vanishes when $\omega \rightarrow 0$, and changes between different interface locations. The difference is more pronounced across the second band, specifically between the case of an interface at the middle of phase $a$ and an interface at the middle of phase $b$. Fig. 4(b) displays $|r|^{2}$ across the unit cell at 10, 100 and $300 \frac{\mathrm{rad}}{\mathrm{s}}$. We observe that the reflection dependency on the interface location becomes greater as the frequency increases.

We recall that in calculating $\tilde{\rho}$ and $\tilde{B}$, and in turn $r$, the right Brillouin zone should be chosen. To demonstrate it in this problem, we plot in Fig. 5 the normalized reflected energy of the exemplary beam, when it is in contact with its homogenized equivalent at $x=0$. We evaluate $|r|^{2}$ across the frequency range of the first two bands, when the interface is located at the middle of phase $a$. In Fig. 5(a), the calculation was carried out using solutions in the $1^{\text {st }}$ Brillouin zone, while Fig. 5(b) uses solutions in the $2^{\text {nd }}$ Brillouin zone for the second band range. The reflected energy is found to be independent of whether we choose the positive normalized wavenumber or its negative value. 
(a)

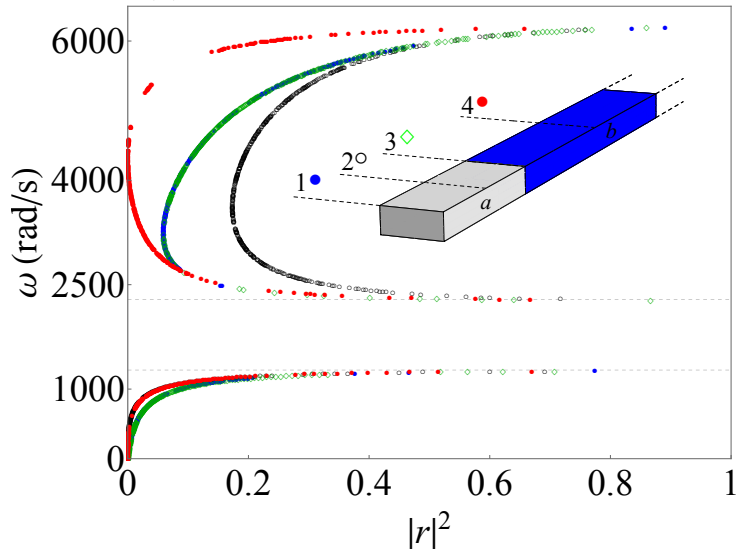

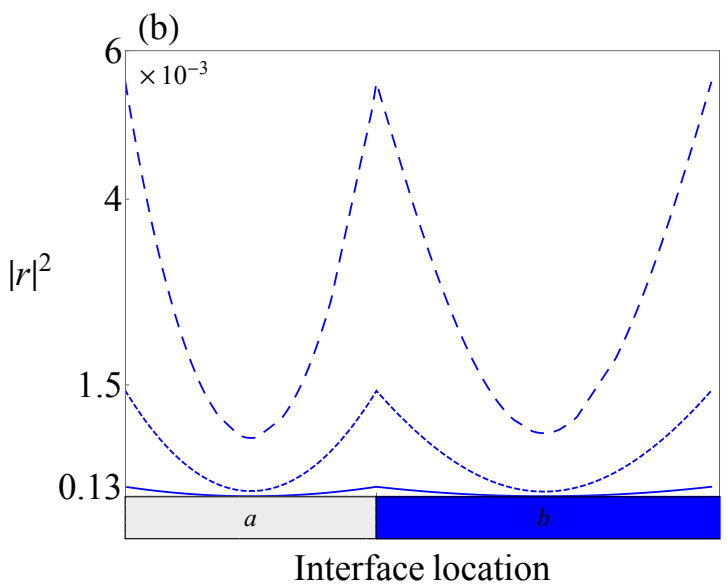

Interface location

Figure 4: (a) Frequency-reflected energy diagram for four locations of the interface with the semi-infinite homogenized beam. (b) Reflected energy as function of the interface location at 10, 100 and $300 \frac{\mathrm{rad}}{\mathrm{s}}$ (solid, dotted and dashed blue curves, respectively).

We observe that calculating $r$ across the second band using $k_{B} l$ in the $1^{\text {st }}$ Brillouin zone violets the conservation of energy, i.e., $|r|^{2}>1$. By contrast, choosing solutions in the $2^{\text {nd }}$ Brillouin zone leads to $|r|^{2}<1$, as it physically should. These results agree with our discussion following Fig. 2, as well as Srivastava and Nemat-Nasser (2014) observation, that certain homogenization schemes for Bloch waves must use proper Brillouin zones.

Finite beam bounded by two semi-infinite homogeneous beams. Next, we consider a composite comprising a finite number of unit cells, and compare its transmission spectrum with the spectrum of a homogeneous beam of the same length, whose properties are the composite homogenized properties, see Fig. 1(d). Fig. 6 shows the normalized transmitted energy through two semi-infinite $b$ phases when they are connected by a finite periodic beam (solid black curves), and when connected by the homogenized beam of the same length (dashed blue curves). In Fig. 6(a), the calculation was carried out for an intermediate beam comprising 13 unit cells, while Fig. 6(b) depicts the result for an intermediate beam comprising 20 unit cells. At low frequencies, the homogenized model reproduces almost identically the transmission characteristics of the periodic beam. For instance, the peak frequencies differ in less than $2 \%$ in the range $0-200 \frac{\mathrm{rad}}{\mathrm{s}}$ for the shorter beams. For longer beams, the difference is even smaller; across the same frequency range, the minimal values of the transmitted energy differ in less than 1.3\%. Across the frequency range of the second band, the homogenized model still reasonably recovers peak locations. However, the error in predicting the minimal transmitted energy becomes substantial, e.g., in the second band depicted in panel (b), the 
(a)

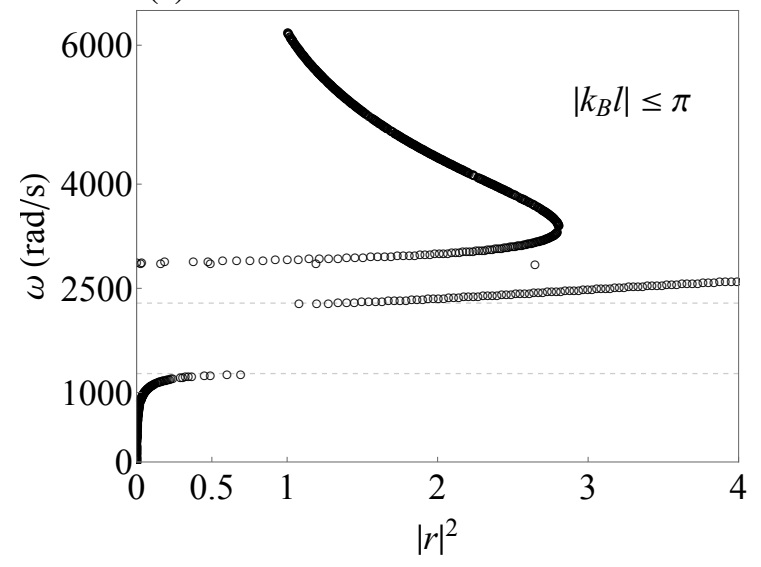

(b)

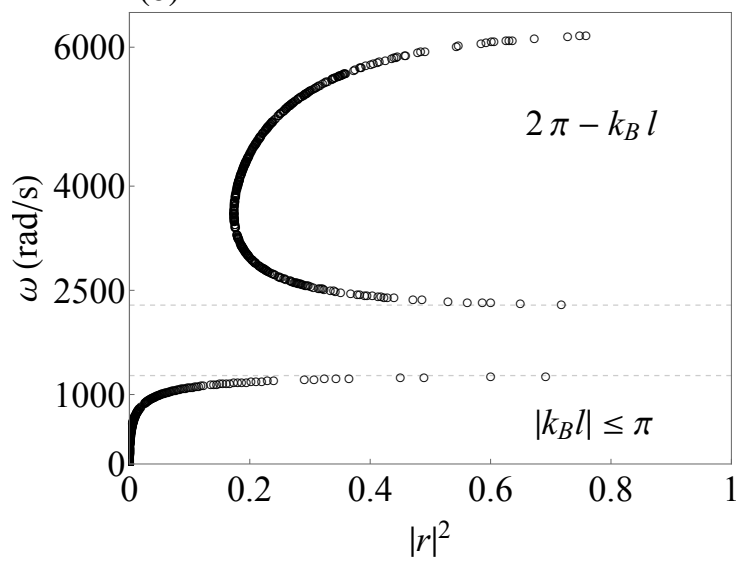

Figure 5: Normalized reflected energy from the interface between the exemplary semi-infinite periodic beam and its homogenized equivalent. Panel (a) uses solutions in the $1^{\text {st }}$ Brillouin zone and for both branches. Panel (b) uses solutions in the $2^{\text {nd }}$ Brillouin zone for the second branch.

highest value of this error is $87 \%$.

\subsection{Homogenization of locally resonant beams}

Next, we apply our scheme to a uniform aluminum beam with periodically attached local resonators. The beam properties are $\rho=2700 \frac{\mathrm{kg}}{\mathrm{m}^{3}}, E=70 \times 10^{9} \mathrm{~Pa}$ and the cross-section of the beam (width $\times$ thickness) is $0.03 \times 0.025 \mathrm{~m}^{2}$. The local resonators properties are $k_{s}=1.455 \times 10^{6} \frac{\mathrm{N}}{\mathrm{m}}$, $m=0.069 \mathrm{~kg}$, as in Xiao et al. (2013); the distance between the resonators is $l=0.04 \mathrm{~m}$. These values correspond to a resonance frequency of $\omega_{0}=4582.8 \frac{\mathrm{rad}}{\mathrm{s}}$.

Dispersion relation and effective properties. Firstly, we evaluate in Fig. 7(a) the exact dispersion relation (continuous curves) using Eq. (8). Subsequently, we calculate $\tilde{\hat{\rho}}$ and the homogenized dispersion relation using Eqs. (17) and (27), when the Fourier expansion comprises 40 terms. The effective mass density, normalized by $\rho$, is depicted in Fig. 7(b), where black circle marks correspond to its real part and red diamond marks correspond to its imaginary part. Note that in this case we used $\left|k_{B} l\right| \leq \pi$; the corresponding homogenized dispersion relation is depicted by circle (real part) and diamond (imaginary part) marks in Fig. 7(a), and demonstrates an excellent agreement with the exact relation. Both relations exhibit a locally resonant gap across the range $4582.8 \frac{\mathrm{rad}}{\mathrm{s}}<\omega<6242.2 \frac{\mathrm{rad}}{\mathrm{s}}$. Note that the frequency at which the gap opens is independent of $l$, 

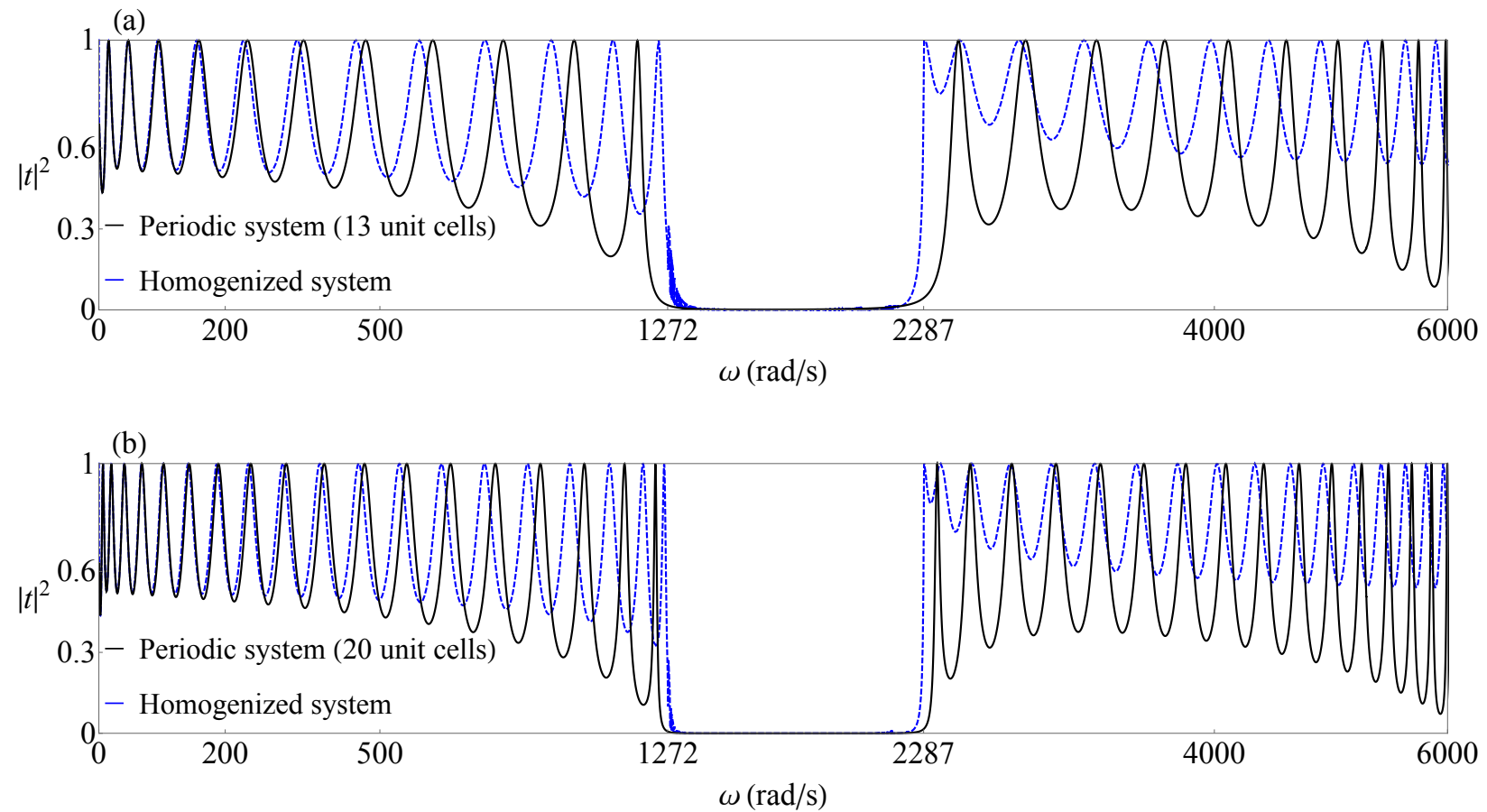

Figure 6: Normalized transmitted energy through two semi-infinite $b$ phases connected by a finite periodic beam (solid black curves), and when connected by the homogenized beam of the same length (dashed blue curves). The length of the intermediate beam in panels (a) and (b) is of 13 and 20 unit cells, respectively.
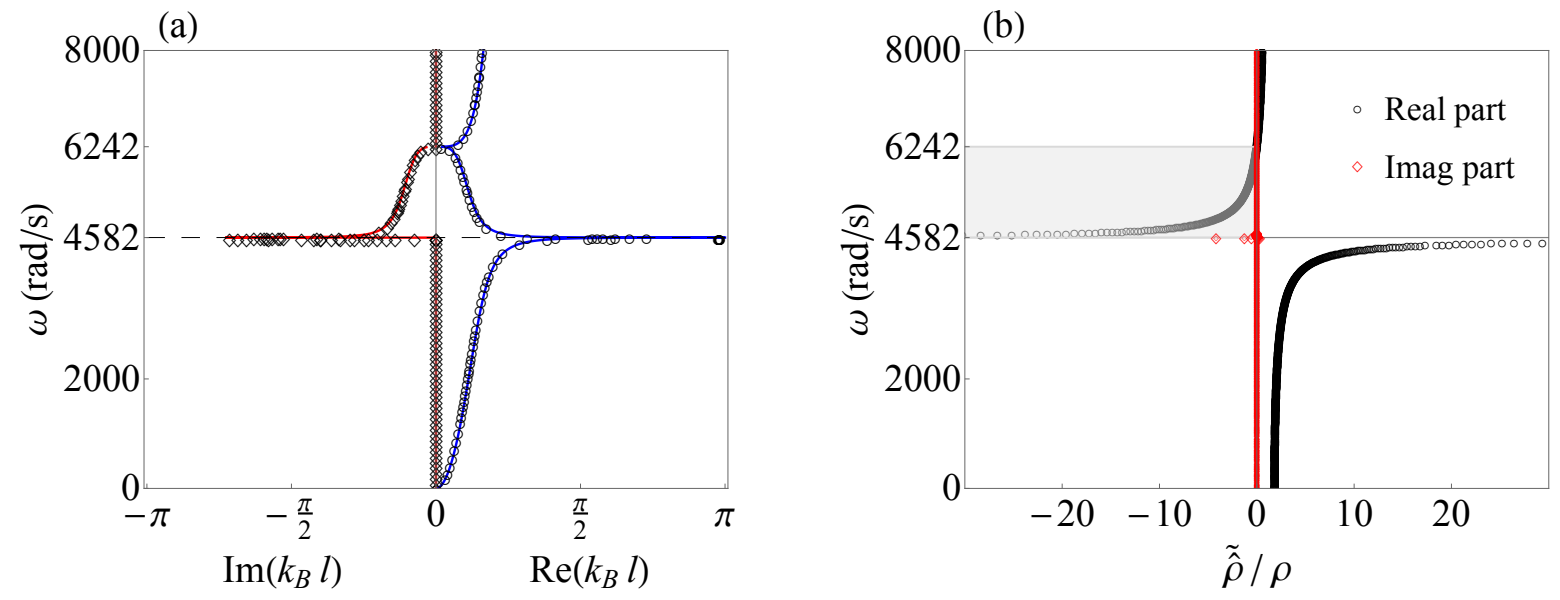

Figure 7: (a) Band structure of the uniform beam with local resonators. The blue (resp. red) curve corresponds to the real (resp. imaginary) part evaluated from the exact relation (8). Circle (resp. diamond) marks correspond to the real (resp. imaginary) part evaluated from the homogenized dispersion relation (17). (b) Normalized effective mass density. 

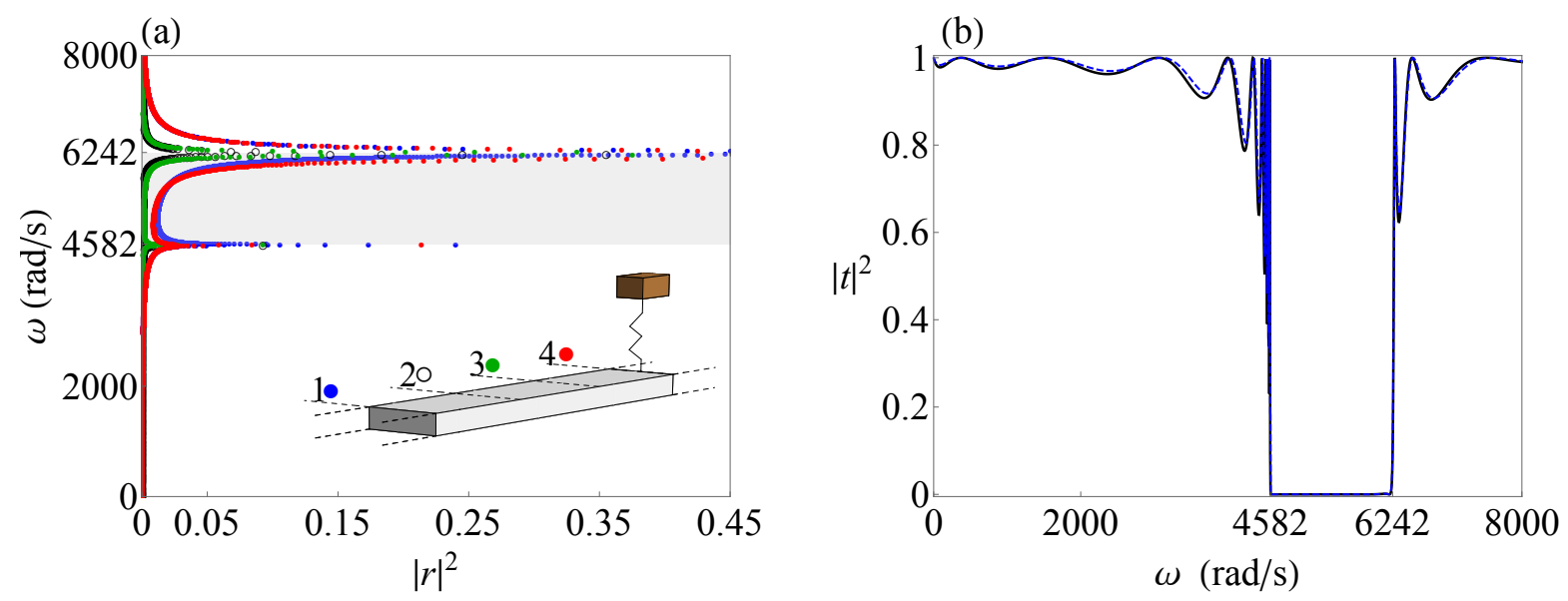

Figure 8: (a) Frequency-reflected energy diagram for four equi-spaced locations of the interface with the semi-infinite homogenized beam. The dashed area indicates the metamaterial region, in which the effective mass density is negative. (b) Normalized transmitted energy through two semi-infinite beams made of aluminum, connected by a locally resonant beam comprising 20 unit cells (solid black curve), and when connected by a homogenized beam of the same length (dashed blue curve).

a known feature of locally resonant gaps.

Contiguous semi-infinite beams. Fig. 8(a) shows the normalized reflected energy of the interface problem between the exemplary semi-infinite beam with local resonators and its semi-infinite homogenized beam for four equi-spaced interface locations, illustrated in the inset. We observe a dependency of the reflected energy with the interface location which is greater in the vicinity of the frequencies at which $\tilde{\hat{\rho}}=0$ and $\infty$. At these frequencies, the difference in the reflected energy is most pronounced between the case of an interface located at one of the ends of the unit cell and an interface near its center. We observe that the homogenized model of the resonant system is less sensitive to the interface location than the model for the composite beam. We further observe that except in the vicinity of the frequencies at which $\tilde{\hat{\rho}}=0$ and $\infty$, the normalized reflected energy vanishes, indicating that the applied homogenization scheme is a valid approximation across a wide range of frequencies. This observation - that locally resonant media are approximated better than non-resonant media by homogenization-was noted before, e.g., by Srivastava and Nemat-Nasser (2014), and Srivastava (2015).

Finite beam bounded by two semi-infinite homogeneous beams. Fig. 8(b) shows the normalized transmitted energy through two semi-infinite beams made of aluminum, connected by a locally resonant beam comprising 20 unit cells (solid black curve), and when connected by a homogenized beam of the same length (dashed blue curve). Remarkably, the homogenized model reproduces almost identically the transmission of the periodic beam. Here again, we find that the homoge- 
nization of the locally resonant medium reproduces the periodic medium characteristics better the characteristics of the the composite medium.

\section{Summary}

We have developed a new homogenized model for composite beams and systems with periodically attached local resonators undergoing flexural motions, based on the approach of Nemat-Nasser and Srivastava (2011). Specifically, we derived macroscopic equations-which take the same form of the local ones - and effective properties, which produce together the exact dispersion relation, as desired. In addition to the simplicity of our homogenization scheme, it does not require the knowledge of local fields; these are actually extractable from it, if wanted.

We have investigated the capability of the homogenized model to capture the dynamic characteristics of the periodic systems, through its application in three numerical settings. Firstly, we have compared the exact dispersion relation of infinite exemplary composite and locally resonant systems with our homogenized model, to find an excellent agreement. Secondly, we studied the reflected energy of an incident wave from a semi-infinite homogenized medium to its semi-infinite periodic counterpart. We showed that at low frequencies, there is no reflection from the media common interface, namely, in this limit our model is able to appropriately match the periodic system impedance. As frequency increases, impedances cannot be matched, and reflection occur in a manner that depends on microscale details, namely, the impedance and length of the phase that borders with the homogenized medium. We observed that this dependency is weaker in the locally resonant case. Finally, we have analyzed the transmitted energy of an incident wave through a finite medium bounded between two semi-infinite homogeneous beams. Specifically, we have compared the transmitted energy when the intermediate medium is periodic, with the transmitted energy when the periodic medium it is replaced by its homogenized equivalent. We observed that the homogenized model of the composite beam neatly captures the first gap, as well as the transmission spectrum across the first band, with some deterioration towards its end. Across the second band, the homogenized model predicts well frequencies of complete transmission, while substantially overestimating magnitudes of minimal transmission. The homogenized approximation for the locally resonant beam is significantly better, having its spectrum almost indistinguishable from the periodic medium spectrum across the two bands. This observation, together with a similar observation in the case of semi-infinite media, suggests that locally resonant systems lend themselves to homogenization better than composite systems, as noted by Srivastava and Nemat-Nasser (2014). It is imperative to extend our analysis to other structural models, such as plates (Antonakakis and Craster, 2012) and torsional systems (Carta and Brun, 2015); this will be pursued in future work. 


\section{Acknowledgements}

We acknowledge the supports of the Israel Science Foundation, funded by the Israel Academy of Sciences and Humanities (Grant no. 1912/15), and the United States-Israel Binational Science Foundation (Grant no. 2014358).

\section{Appendix A}

Within a homogeneous phase, the state vector $\mathrm{s}(x)=\{u(x), \theta(x), M(x), V(x)\}^{\top}$ can be expressed in the matrix form

$$
\mathrm{s}(x)=\left[\begin{array}{cccc}
e^{i k x} & e^{-k x} & e^{-i k x} & e^{k x} \\
i k e^{i k x} & -k e^{-k x} & -i k e^{-i k x} & k e^{k x} \\
-B k^{2} e^{i k x} & B k^{2} e^{-k x} & -B k^{2} e^{-i k x} & B k^{2} e^{k x} \\
i B k^{3} e^{i k x} & B k^{3} e^{-k x} & -i B k^{3} e^{-i k x} & -B k^{3} e^{k x}
\end{array}\right] \cdot\left\{\begin{array}{c}
C^{+} \\
D^{+} \\
C^{-} \\
D^{-}
\end{array}\right\}
$$

where $k=\sqrt[4]{\rho A \omega^{2} / B}$ and the coefficients $C^{ \pm}, D^{ \pm}$represent amplitudes of corresponding waves. Commonly, the state vector in periodic systems, and in turn the dispersion relation, are expressed in terms of the transfer matrix. However, in certain cases the transfer matrix formulation is prone to numerical instabilities (Dunkin, 1965, Pérez-Álvarez and García-Moliner, 2004). Therefore, we used a formulation based on the numerically stable hybrid matrix (Tan, 2006, Pérez-Álvarez et al., 2015, Shmuel and Pernas-Salomón, 2016), as follow. We define a modified state vector 
$\mathrm{s}_{\mathrm{m}}(x)=\left\{u(x), \theta(x) k^{(a)^{-1}}, M(x) k^{(a)^{-2}}, V(x) k^{(a)^{-3}}\right\}^{\top}$, for which the hybrid matrix reads in Eq. (6)

$$
\begin{aligned}
& \mathrm{H}^{(i)}=\left[\begin{array}{cccc}
1 & 1 & e^{i k^{(i)} l^{(i)}} & e^{-k^{(i)} l^{(i)}} \\
i \frac{k^{(i)}}{k^{(a)}} & -\frac{k^{(i)}}{k^{(a)}} & -i \frac{k^{(i)}}{k^{(a)}} e^{i k^{(i)} l^{(i)}} & \frac{k^{(i)}}{k^{(a)}} e^{-k^{(i)} l^{(i)}} \\
-B^{(i)}\left(\frac{k^{(i)}}{k^{(a)}}\right)^{2} e^{i k^{(i)} l^{(i)}} & B^{(i)}\left(\frac{k^{(i)}}{k^{(a)}}\right)^{2} e^{-k^{(i)} l^{(i)}} & -B^{(i)}\left(\frac{k^{(i)}}{k^{(a)}}\right)^{2} & B^{(i)}\left(\frac{k^{(i)}}{k^{(a)}}\right)^{2} \\
i B^{(i)}\left(\frac{k^{(i)}}{k^{(a)}}\right)^{3} e^{i k^{(i)} l^{(i)}} & B^{(i)}\left(\frac{k^{(i)}}{k^{(a)}}\right)^{3} e^{-k^{(i)} l^{(i)}} & -i B^{(i)}\left(\frac{k^{(i)}}{k^{(a)}}\right)^{3} & -B^{(i)}\left(\frac{k^{(i)}}{k^{(a)}}\right)^{3}
\end{array}\right]
\end{aligned}
$$

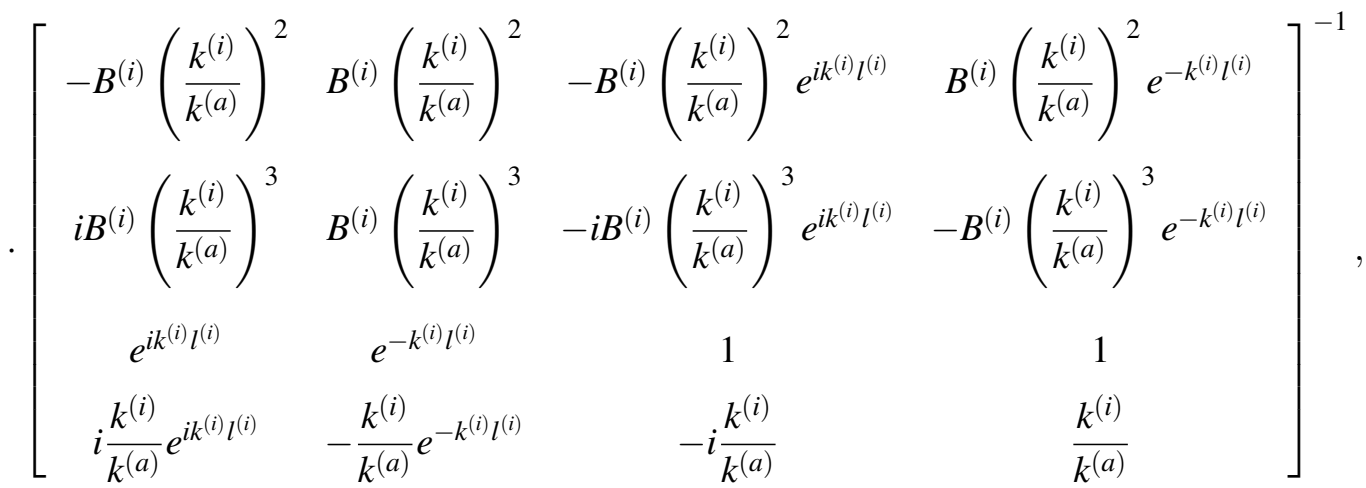

where $B^{(i)}$ denotes the bending stiffness of the phase $i$, and the $k^{(i)}$ (resp. $k^{(a)}$ ) is the value that $k$ takes in the phase $i$ (resp. $a$ ). The matrix elements of $\mathrm{H}^{(i)}$ are

$$
\begin{aligned}
& \mathrm{h}_{11}^{(i)}=\left(\frac{k^{(a)}}{k^{(i)}}\right)^{2} \frac{\sin l^{(i)} k^{(i)} \sinh l^{(i)} k^{(i)}}{B^{(i)} \cos l^{(i)} k^{(i)} \cosh l^{(i)} k^{(i)}+B^{(i)}}, \\
& \mathrm{h}_{12}^{(i)}=\left(\frac{k^{(a)}}{k^{(i)}}\right)^{3} \frac{\cos l^{(i)} k^{(i)} \sinh l^{(i)} k^{(i)}-\sin l^{(i)} k^{(i)} \cosh l^{(i)} k^{(i)}}{B^{(i)} \cos l^{(i)} k^{(i)} \cosh l^{(i)} k^{(i)}+B^{(i)}}, \\
& \mathrm{h}_{13}^{(i)}=\frac{\cos l^{(i)} k^{(i)}+\cosh l^{(i)} k^{(i)}}{\cos l^{(i)} k^{(i)} \cosh l^{(i)} k^{(i)}+1}, \\
& \mathrm{~h}_{14}^{(i)}=-\frac{k^{(a)}}{k^{(i)}} \frac{\sin l^{(i)} k^{(i)}+\sinh l^{(i)} k^{(i)}}{\cos l^{(i)} k^{(i)} \cosh l^{(i)} k^{(i)}+1},
\end{aligned}
$$




$$
\begin{aligned}
\mathrm{h}_{21}^{(i)} & =-\frac{k^{(a)}}{k^{(i)}} \frac{\cos l^{(i)} k^{(i)} \sinh l^{(i)} k^{(i)}+\sin l^{(i)} k^{(i)} \cosh l^{(i)} k^{(i)}}{B^{(i)} \cos l^{(i)} k^{(i)} \cosh l^{(i)} k^{(i)}+B^{(i)}} \\
\mathrm{h}_{22}^{(i)} & =\mathrm{h}_{11}^{(i)}, \\
\mathrm{h}_{23}^{(i)} & =\frac{k^{(i)}}{k^{(a)}} \frac{\sin l^{(i)} k^{(i)}-\sinh l^{(i)} k^{(i)}}{\cos l^{(i)} k^{(i)} \cosh l^{(i)} k^{(i)}+1} \\
\mathrm{~h}_{24}^{(i)} & =\mathrm{h}_{13}^{(i)},
\end{aligned}
$$

$$
\begin{aligned}
& \mathrm{h}_{31}^{(i)}=\mathrm{h}_{13}^{(i)}, \\
& \mathrm{h}_{32}^{(i)}=\mathrm{h}_{14}^{(i)}, \\
& \mathrm{h}_{33}^{(i)}=\left(\frac{k^{(i)}}{k^{(a)}}\right)^{2} \frac{B^{(i)} \sin l^{(i)} k^{(i)} \sinh l^{(i)} k^{(i)}}{\cos l^{(i)} k^{(i)} \cosh l^{(i)} k^{(i)}+1}, \\
& \mathrm{~h}_{34}^{(i)}=\frac{k^{(i)}}{k^{(a)}} \frac{B^{(i)}\left[\cos l^{(i)} k^{(i)} \sinh l^{(i)} k^{(i)}-\sin l^{(i)} k^{(i)} \cosh l^{(i)} k^{(i)}\right]}{\cos l^{(i)} k^{(i)} \cosh l^{(i)} k^{(i)}+1},
\end{aligned}
$$

$$
\begin{aligned}
& \mathrm{h}_{41}^{(i)}=\mathrm{h}_{23}^{(i)}, \\
& \mathrm{h}_{42}^{(i)}=\mathrm{h}_{13}^{(i)} \\
& \mathrm{h}_{43}^{(i)}=-\left(\frac{k^{(i)}}{k^{(a)}}\right)^{3} \frac{B^{(i)}\left[\cos l^{(i)} k^{(i)} \sinh l^{(i)} k^{(i)}+\sin l^{(i)} k^{(i)} \cosh l^{(i)} k^{(i)}\right]}{\cos l^{(i)} k^{(i)} \cosh l^{(i)} k^{(i)}+1} \\
& \mathrm{~h}_{44}^{(i)}=\mathrm{h}_{33}^{(i)}
\end{aligned}
$$

The total hybrid matrix corresponding to a stack comprising $i$ phases, $\mathrm{H}^{(1, i)}$, is determined in terms of the total hybrid matrix of the first $i-1$ phases, $\mathrm{H}^{(1, i-1)}$, and the hybrid matrix of the $i$ th phase, 
$\mathrm{H}^{(i)}$, as follows

$$
\begin{aligned}
& \mathrm{H}_{22}^{(1, i)}=\mathrm{H}_{22}^{(i)}+\mathrm{H}_{21}^{(i)} \cdot\left[\mathrm{I}_{2}-\mathrm{H}_{22}^{(1, i-1)} \cdot \mathrm{H}_{11}^{(i)}\right]^{-1} \cdot \mathrm{H}_{22}^{(1, i-1)} \cdot \mathrm{H}_{12}^{(i)}, \\
& \mathrm{H}_{21}^{(1, i)}=\mathrm{H}_{21}^{(i)} \cdot\left[\mathrm{I}_{2}-\mathrm{H}_{22}^{(1, i-1)} \cdot \mathrm{H}_{11}^{(i)}\right]^{-1} \cdot \mathrm{H}_{21}^{(1, i-1)}, \\
& \mathrm{H}_{12}^{(1, i)}=\mathrm{H}_{12}^{(1, i-1)} \cdot \mathrm{H}_{12}^{(i)}+\mathrm{H}_{12}^{(1, i-1)} \cdot \mathrm{H}_{11}^{(i)} \cdot\left[\mathrm{I}_{2}-\mathrm{H}_{22}^{(1, i-1)} \cdot \mathrm{H}_{11}^{(i)}\right]^{-1} \cdot \mathrm{H}_{22}^{(1, i-1)} \cdot \mathrm{H}_{12}^{(i)}, \\
& \mathrm{H}_{11}^{(1, i)}=\mathrm{H}_{11}^{(1, i-1)}+\mathrm{H}_{12}^{(1, i-1)} \cdot \mathrm{H}_{11}^{(i)} \cdot\left[\mathrm{I}_{2}-\mathrm{H}_{22}^{(1, i-1)} \cdot \mathrm{H}_{11}^{(i)}\right]^{-1} \cdot \mathrm{H}_{21}^{(1, i-1)},
\end{aligned}
$$

where $\mathrm{H}_{11}, \mathrm{H}_{12}, \mathrm{H}_{21}, \mathrm{H}_{22}$ denote the $2 \times 2$ sub-blocks of the corresponding hybrid matrix, and $\mathrm{I}_{2}$ denotes the unit matrix of the same order.

\section{References}

Alireza V. Amirkhizi. Homogenization of layered media based on scattering response and field integration. Mechanics of Materials, 114:76 - 87, 2017. ISSN 0167-6636. doi: https://doi.org/ 10.1016/j.mechmat.2017.06.008. URL http: / / www. sciencedirect.com/science/ article/pii/s0167663617302016.

T. Antonakakis and R. V. Craster. High-frequency asymptotics for microstructured thin elastic plates and platonics. Proceedings of the Royal Society of London A: Mathematical, Physical and Engineering Sciences, 468(2141):1408-1427, 2012. ISSN 1364-5021. doi: 10.1098/rspa.2011. 0652. URL http: / / rspa.royalsocietypublishing.org/content/468/2141/ 1408.

E. G. Barnwell, W. J. Parnell, and I. D. Abrahams. Tunable elastodynamic band gaps. Extreme Mechanics Letters, 12:23-29, 2017. ISSN 2352-4316. doi: http://doi.org/10.1016/j.eml. 2016.10.009. URL http://wWw.sciencedirect.com/science/article/pii/ S2352431616300815. Frontiers in Mechanical Metamaterials.

D. Bigoni, S. Guenneau, A. B. Movchan, and M. Brun. Elastic metamaterials with inertial locally resonant structures: Application to lensing and localization. Phys. Rev. B, 87:174303, May 2013. doi: 10.1103/PhysRevB.87.174303. URL https : / / i ink aps . org/doi/10 . 1103 /PhysRevB.87.174303.

F. Bloch. Z. Physik, 52:555, 1929. doi: https://doi.org/10.1007/BF01339455. 
G. Carta and M. Brun. Bloch-floquet waves in flexural systems with continuous and discrete elements. Mechanics of Materials, 87:11-26, 2015. ISSN 0167-6636. doi: http://dx.doi.org/ 10.1016/j.mechmat.2015.03.004. URL http://www.sciencedirect.com/science/ article/pii/s0167663615000678.

P. Celli and S. Gonella. Low-frequency spatial wave manipulation via phononic crystals with relaxed cell symmetry. Journal of Applied Physics, 115(10):103502, 2014. doi: 10.1063/1. 4867918. URL https://doi.org/10.1063/1.4867918.

V. Cerdán-Ramírez, B. Zenteno-Mateo, M. P. Sampedro, M. A. Palomino-Ovando, B. FloresDesirena, and F. Pérez-Rodríguez. Anisotropy effects in homogenized magnetodielectric photonic crystals. Journal of Applied Physics, 106(10):103520, 2009. doi: 10.1063/1.3261758. URL https://doi.org/10.1063/1.3261758.

Y. Chen, G. Hu, and G. Huang. A hybrid elastic metamaterial with negative mass density and tunable bending stiffness. Journal of the Mechanics and Physics of Solids, 105:179 - 198, 2017. ISSN 0022-5096. doi: https://doi.org/10.1016/j.jmps.2017.05.009. URL http://www . sciencedirect.com/science/article/pii/s0022509617301229.

D.J. Colquitt, M. Brun, M. Gei, A.B. Movchan, N.V. Movchan, and I.S. Jones. Transformation elastodynamics and cloaking for flexural waves. Journal of the Mechanics and Physics of Solids, 72:131-143, 2014. ISSN 0022-5096. doi: http://dx.doi.org/10.1016/j.jmps. 2014.07.014. URL http://www.sciencedirect.com/science/article/pii/ S0022509614001586.

R. V. Craster, J. Kaplunov, and A. V. Pichugin. High-frequency homogenization for periodic media. Proceedings of the Royal Society of London A: Mathematical, Physical and Engineering Sciences, 466(2120):2341-2362, 2010. ISSN 1364-5021. doi: 10.1098/rspa.2009.0612. URL http://rspa.royalsocietypublishing.org/content/466/2120/2341.

J. W. Dunkin. Computation of modal solutions in layered, elastic media at high frequencies. Bulletin of the Seismological Society of America, 55(2):335, 1965. URL +http://dx.doi. org/.

F. Farzbod and M. J. Leamy. Analysis of bloch's method and the propagation technique in periodic structures. Journal of Vibration and Acoustics, 133(3):031010, 2011. doi: http://dx.doi.org/10. 1115/1.4003202. URL http://dx.doi.org/10.1115/1.4003202.

K.F. Graff. Wave Motion in Elastic Solids. Dover Books on Physics Series. Dover Publications, 1975. ISBN 9780486667454. URL https://books.google.co.il/books?id= 5 cZFRwLuhdQC. 
Z. Hashin. Analysis of composite materials-a survey. Journal of Applied Mechanics, 50(3):481505, September 1983. ISSN 0021-8936. doi: 10.1115/1.3167081. URL http://dx.doi. org/10.1115/1.3167081.

H. Herzig Sheinfux, I. Kaminer, Y. Plotnik, G. Bartal, and M. Segev. Subwavelength multilayer dielectrics: Ultrasensitive transmission and breakdown of effective-medium theory. Phys. Rev. Lett., 113:243901, Dec 2014. doi: 10.1103/PhysRevLett.113.243901. URL https: / / I ink. aps.org/doi/10.1103/PhysRevLett.113.243901.

L.M. Joseph and R.V. Craster. Reflection from a semi-infinite stack of layers using homogenization. Wave Motion, 54:145 - 156, 2015. ISSN 0165-2125. doi: https://doi.org/10.1016/j.wavemoti. 2014.12.003. URL http://www.sciencedirect.com/science/article/pii/ S0165212514001747.

C. Kittel. Introduction to solid state physics, 8th ed. John Wiley \& Sons, Inc., 2005.

Jan Korvink and Oliver Paul. MEMS: A Practical Guide of Design, Analysis, and Applications. Springer-Verlag Berlin Heidelberg, 2006. ISBN 978-3-540-21117-4. doi: http://dx.doi.org/10. 1007/978-3-540-33655-6.

G. Ma and P. Sheng. Acoustic metamaterials: From local resonances to broad horizons. Science Advances, 2(2), 2016. doi: 10.1126/sciadv.1501595. URL http: / / advances . sciencemag . org/content/2/2/e1501595.

G. W. Milton and J. R. Willis. On modifications of newton's second law and linear continuum elastodynamics. Proceedings of the Royal Society of London A: Mathematical, Physical and Engineering Sciences, 463(2079):855-880, 2007. ISSN 1364-5021. doi: 10.1098/rspa.2006. 1795. URL http://rspa.royalsocietypublishing.org/content/463/2079/ 855.

G.W. Milton. The Theory of Composites, volume 6 of Cambridge Monographs on Applied and Computational Mathematics. Cambridge University Press, New York, 2002.

D. Misseroni, D. J. Colquitt, A. B. Movchan, N. V. Movchan, and I. S. Jones. Cymatics for the cloaking of flexural vibrations in a structured plate. Scientific Reports, 6:23929 EP -, 042016. URL http://dx.doi.org/10.1038/srep23929.

S. Nemat-Nasser and M. Hori. Micromechanics : overall properties of heterogeneous materials, Second edition. Amsterdam: Elsevier, 1999. ISBN 0444500847. 
S. Nemat-Nasser and A. Srivastava. Overall dynamic constitutive relations of layered elastic composites. Journal of the Mechanics and Physics of Solids, 59(10):1953-1965, 2011. ISSN 0022-5096. doi: http://dx.doi.org/10.1016/j.jmps.2011.07.008. URL http://www . sciencedirect.com/science/article/pii/s0022509611001475.

S. Nemat-Nasser, J. R. Willis, A. Srivastava, and A. V. Amirkhizi. Homogenization of periodic elastic composites and locally resonant sonic materials. Phys. Rev. B, 83:104103, Mar 2011. doi: 10.1103/PhysRevB.83.104103. URL https://link.aps.org/doi/10 . 1103 /PhysRevB.83.104103.

M. Ostoja-Starzewski. Lattice models in micromechanics. Applied Mechanics Reviews, 55(1): 35-60, 01 2002. URL http://dx.doi.org/10.1115/1.1432990.

R. Pérez-Álvarez and F. García-Moliner. Transfer Matrix, Green Function and related techniques: Tools for the study of multilayer heterostructures. Universitat Jaume I, Castellón de la Plana, Spain, 2004. ISBN 84-8021-474-4.

R. Pérez-Álvarez, R. Pernas-Salomón, and V.R. Velasco. Relations between transfer matrices and numerical stability analysis to avoid the $\omega \mathrm{d}$ problem. SIAM Journal on Applied Mathematics, 75(4):1403-1423, 2015. doi: 10.1137/140993442. URL http://dx.doi.org/10.1137/ 140993442.

G. Shmuel and R. Pernas-Salomón. Manipulating motions of elastomer films by electrostaticallycontrolled aperiodicity. Smart Materials and Structures, 25(12):125012, 2016. URL http: //stacks.iop.org/0964-1726/25/i=12/a=125012.

Z. Shuguang, N. Tianxin, W. Xudong, and F. Jialu. Studies of band gaps in flexural vibrations of a locally resonant beam with novel multi-oscillator configuration. Journal of Vibration and Control, 23(10):1663-1674, 2018/03/08 2015. doi: 10.1177/1077546315598032. URL https: //doi.org/10.1177/1077546315598032.

A. Srivastava. Elastic metamaterials and dynamic homogenization: a review. International Journal of Smart and Nano Materials, 6(1):41-60, 2015. doi: 10.1080/19475411.2015.1017779. URL https://doi.org/10.1080/19475411.2015.1017779.

A. Srivastava and S. Nemat-Nasser. On the limit and applicability of dynamic homogenization. Wave Motion, 51(7):1045 - 1054, 2014. ISSN 0165-2125. doi: https://doi.org/ 10.1016/j.wavemoti.2014.04.003. URL http://www.sciencedirect.com/science/ article/pii/s0165212514000614. 
X. Sun, C. Zhou, M. Ichchou, J.-P. Lainé, and A.-M. Zine. Multi-scale homogenization of transversal waves in periodic composite beams. International Journal of Applied Mechanics, 09(03):1750039, 2017. doi: 10.1142/S1758825117500399. URL http://www . worldscientific.com/doi/abs/10.1142/s1758825117500399.

E. L. Tan. Generalized eigenproblem of hybrid matrix for floquet wave propagation in onedimensional phononic crystals with solids and fluids. Ultrasonics, 50(1):91 - 98, 2010. ISSN 0041-624X. doi: https://doi.org/10.1016/j.ultras.2009.09.007. URL http://www . sciencedirect.com/science/article/pii/s0041624X09001085.

E.L. Tan. Hybrid compliance-stiffness matrix method for stable analysis of elastic wave propagation in multilayered anisotropic media. The Journal of the Acoustical Society of America, 119 (1), 2006. doi: http://dx.doi.org/10.1121/1.2139617. URL http://scitation.aip.org/ content/asa/journal/jasa/119/1/10.1121/1.2139617.

D. Torrent, Y. Pennec, and B. Djafari-Rouhani. Effective medium theory for elastic metamaterials in thin elastic plates. Phys. Rev. B, 90:104110, Sep 2014. doi: 10.1103/PhysRevB.90.104110. URL https://link.aps.org/doi/10.1103/PhysRevB.90.104110.

J.R. Willis. Exact effective relations for dynamics of a laminated body. Mechanics of Materials, 41(4):385 - 393, 2009. ISSN 0167-6636. doi: http://dx.doi.org/10.1016/j.mechmat. 2009.01.010. URL http://www.sciencedirect.com/science/article/pii/ S0167663609000118. The Special Issue in Honor of Graeme W. Milton.

J.R. Willis. Some thoughts on dynamic effective properties-a working document. 2013. URL https://arxiv.org/abs/1311.3875.

Y. Xiao, J. Wen, D. Yu, and X. Wen. Flexural wave propagation in beams with periodically attached vibration absorbers: Band-gap behavior and band formation mechanisms. Journal of Sound and Vibration, 332(4):867 - 893, 2013. ISSN 0022-460X. doi: https://doi.org/10.1016/j.jsv. 2012.09.035. URL http://www.sciencedirect.com/science/article/pii/ S0022460X12007596.

Y. Xu, X. Zhou, W. Wang, L. Wang, F. Peng, and B. Li. On natural frequencies of nonuniform beams modulated by finite periodic cells. Physics Letters A, 380(40):3278 - 3283, 2016. ISSN 0375-9601. doi: https://doi.org/10.1016/j.physleta.2016.07.057. URL http: //www.sciencedirect.com/science/article/pii/s0375960116304960.

W. Yang, B. Kim, S. Cho, and J. Park. Experimental Method to Evaluate Effective Dynamic Properties of a Meta-Structure for Flexural Vibrations. Experimental Mechanics, 57(3):417425, MAR 2017. ISSN 0014-4851. doi: \{10.1007/s11340-016-0242-2\}. 
D. Yu, Y. Liu, G. Wang, H. Zhao, and J. Qiu. Flexural vibration band gaps in timoshenko beams with locally resonant structures. Journal of Applied Physics, 100(12):124901, 2006. doi: 10. 1063/1.2400803. URL https://doi.org/10.1063/1.2400803.

A. Zareei, A. Darabi, M. J. Leamy, and Mohammad-Reza Alam. Continuous profile flexural grin lens: Focusing and harvesting flexural waves. Applied Physics Letters, 112(2):023901, 2018. doi: 10.1063/1.5008576. URL https://doi.org/10.1063/1.5008576. 